\title{
Simultaneous EUS-FNA Diagnosis and TNM Staging of a Pancreatic Neuroendocrine Tumor in a Patient with an Unrecognized MEN Type 1
}

\author{
Francesco Ferrara, ${ }^{1}$ Carmelo Luigiano, ${ }^{1}$ Antonella Maimone, ${ }^{1}$ \\ Marco Bassi, ${ }^{1}$ Anna Maria Polifemo, ${ }^{1}$ Paola Baccarini, ${ }^{2}$ Vincenzo Cennamo, ${ }^{3}$ \\ Nadia Cremonini, ${ }^{4}$ and Carlo Fabbri ${ }^{1}$ \\ ${ }^{1}$ Unit of Gastroenterology and Digestive Endoscopy, Bellaria and Maggiore Hospitals, AUSL Bologna, Largo Nigrisoli 2, \\ 40133 Bologna, Italy \\ ${ }^{2}$ Unit of Pathology, Bellaria Hospital, AUSL Bologna, 40139 Bologna, Italy \\ ${ }^{3}$ Unit of Metropolitan Area Digestive Endoscopy, Bellaria Hospital, AUSL Bologna, 40139 Bologna, Italy \\ ${ }^{4}$ Unit of Endocrinology, Maggiore Hospital, AUSL Bologna, 40139 Bologna, Italy
}

Correspondence should be addressed to Francesco Ferrara, f.ferrara@ausl.bologna.it

Received 9 July 2012; Accepted 12 September 2012

Academic Editors: Y. Aoki and S. B. Chichareon

Copyright (C) 2012 Francesco Ferrara et al. This is an open access article distributed under the Creative Commons Attribution License, which permits unrestricted use, distribution, and reproduction in any medium, provided the original work is properly cited.

We report the case of a woman who, during oncological followup for bronchial carcinoid (diagnosed in 2005), papillary thyroid carcinoma, and bilateral parathyroid adenoma (simultaneously diagnosed in 2007), performed a pancreatic endoscopic ultrasonography with fine needle agobiopsy (EUS-FNA) for a positron emission tomography (PET) suspicion of pancreatic and hepatic lesions; during the procedure, the pancreatic and liver lesions were confirmed, and a peripancreatic lymph node involvement was found, allowing a complete pTNM staging during the same procedure.

\section{Case}

A 48-year-old woman, with no family history of neoplastic diseases, underwent in 2005 to right pulmonary resection for a bronchial carcinoids and was in oncological follow-up for this reason.

In 2007, during a normal physical examination, a $2 \mathrm{~cm}$ sized nodule in her left thyroid lobe was palpated. The thyroid function tests were normal. The ultrasonography report revealed a solid hypoechoic nodule measuring $2.5 \times 1 \mathrm{~cm}$ in the left lobe. A fine needle aspiration was attempted under ultrasound guidance and the final cytodiagnosis was papillary thyroid carcinoma.

Following cytodiagnosis, a total thyroidectomy was performed, but at the time of surgery, during the neck exploration, the patient was found to have bilateral enlargement of parathyroid glands with nodular aspect and all glands were resected. Histology confirmed the diagnosis of thyroid papillary cancer and revealed bilateral parathyroid adenoma.

In September 2008, a biochemical screening revealed high plasma levels of CgA of 2230 (normal 20-150) and serum gastrin $(648 \mathrm{pg} / \mathrm{mL})$. She was then referred to our hospital.

A 68Ga-DOTANOC-PET was performed and revealed 3 small areas of hyperaccumulation in pancreatic region and one in the liver.

For this reason, an EUS was performed and identified 2 nodular hypoechoic lesions of $5 \mathrm{~mm}$ with hypervascular Doppler pattern located in the body and in the tail of the pancreas. During the exploration, also a peripancreatic nodal involvement was found and the suspected metastasis $(15 \mathrm{~mm})$ of left liver was confirmed. We performed EUSguided FNA with a $22 \mathrm{G}$ and a $25 \mathrm{G}$ needle, with onsite cytopathologist, and a Pan-NET (T) tumor (Figure 1) 


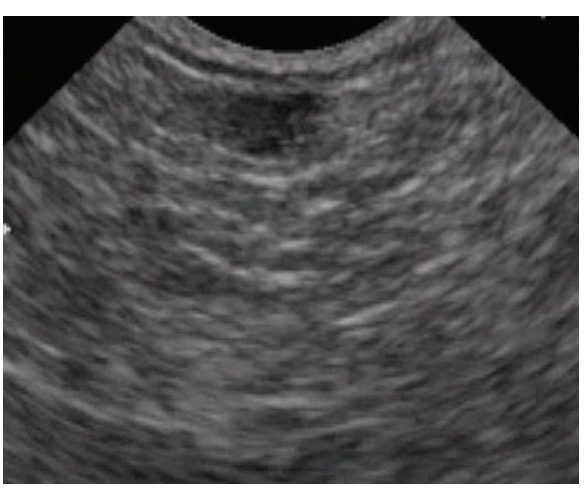

(a)

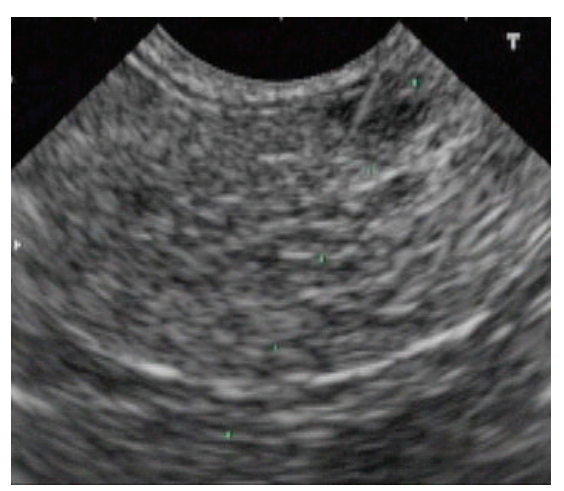

(b)

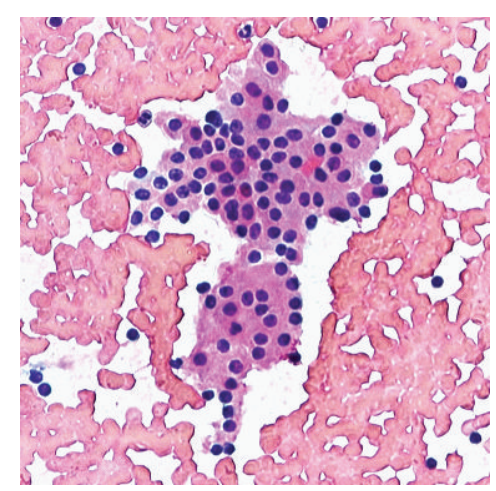

(c)

FIGURE 1: EUS (a), FNA (b), and cytological (c) images of the neuroendocrine pancreatic tumor.

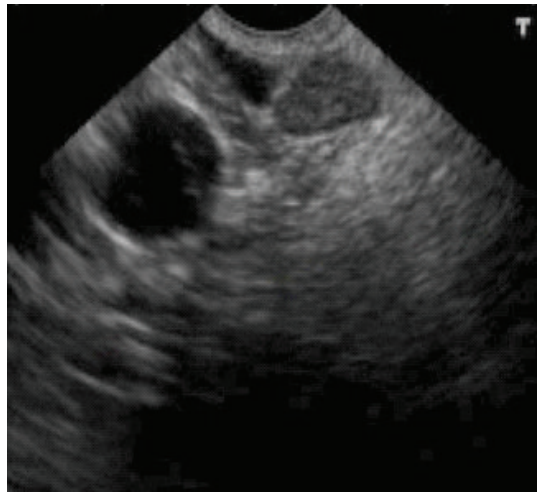

(a)

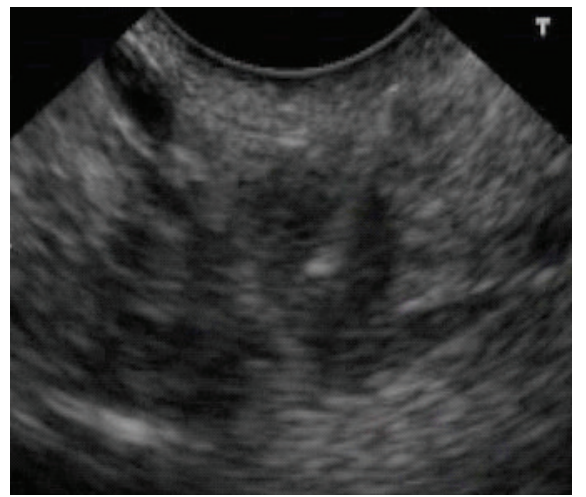

(b)

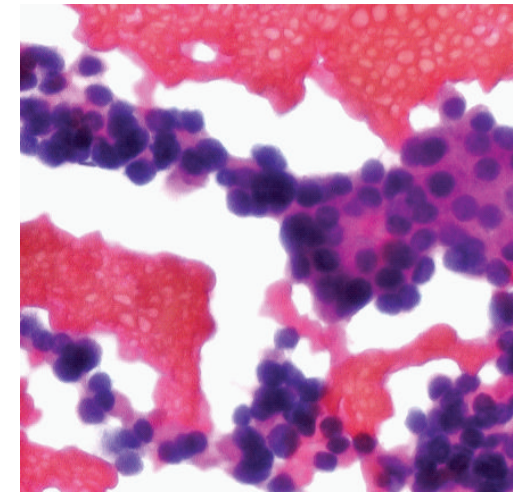

(c)

Figure 2: EUS (a), FNA (b), and cytological (c) images of the lymphonodal metastases.

with (N) lymph node (Figure 2) and (M) liver (Figure 3) involvement was diagnosed.

A mutation of the MEN-1 gene was identified and, due to the high level of serum gastrin, a diagnosis of gastrinoma was reached.

She started therapy with octreotide LAR followed by receptor radiometabolic therapy with radiolabelled somatostatin analogues (177 Lu-DOTATATE) and she is presently alive.

\section{Discussion}

Multiple endocrine neoplasia type 1 (MEN-1) syndrome is a rare disease, inherited as an autosomal dominant trait with an estimated prevalence of 0.01-2.5/100000 [1].

MEN-1 syndrome is characterized by parathyroid hyperplasia, neuroendocrine pancreatoduodenal tumors, and pituitary adenomas. Less commonly, MEN-1 patients can develop bronchial, gastrointestinal, and thymic carcinoids, benign thyroid and adrenocortical tumors, lipomas, angiofibromas, skin collagenomas, and ependymomas of the central nervous system [2].
Thyroid disease can be observed in over $25 \%$ of MEN1 patients, $[3,4]$, and it can be detected incidentally during parathyroid surgery.

Only three cases of papillary thyroid cancer combined with MEN1 were reported in the literature and seems that these cases did not correlate to MEN1 [4-6].

Probably for this reason, the diagnosis of MEN1 was not made before.

About $20 \%$ of MEN-1 patients succumb to malignant tumors and malignant Pan-NET are unequivocally the most frequent cause of death [7].

Imaging modalities such as CT and MRI have enabled detection of Pan-NETs. Overall sensitivity for CT ranges from 64 to $82 \%$, with lesser sensitivity for tumors $<1 \mathrm{~cm}$ in size. Similarly, MRI offers superb imaging of the pancreas, with sensitivity of up to $90 \%$ in Pan-NETs $[8,9]$.

Nuclear medicine studies have been developed to target specific receptors on Pan-NET tumor cells with radiolabeled receptor-binding peptides. The most common of these tests is somatostatin receptor scintigraphy (SRS). The sensitivity of SRS for detecting gastrinomas is as high as 75-100\%; in contrast, SRS is able to detect insulinomas in approximately only half of the times [10]. 


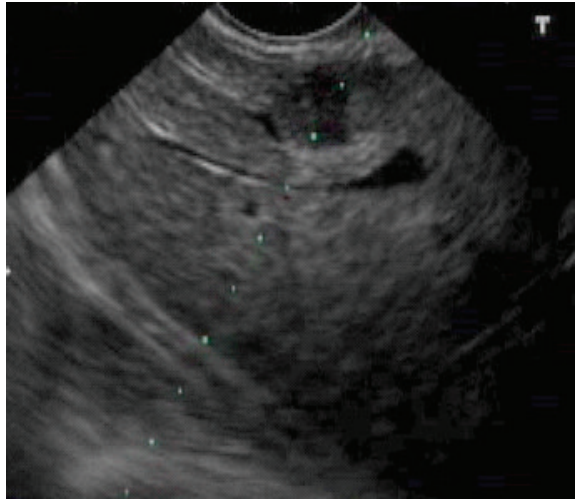

(a)

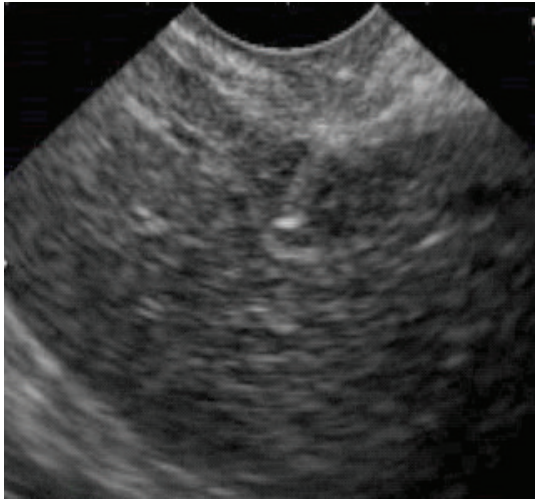

(b)

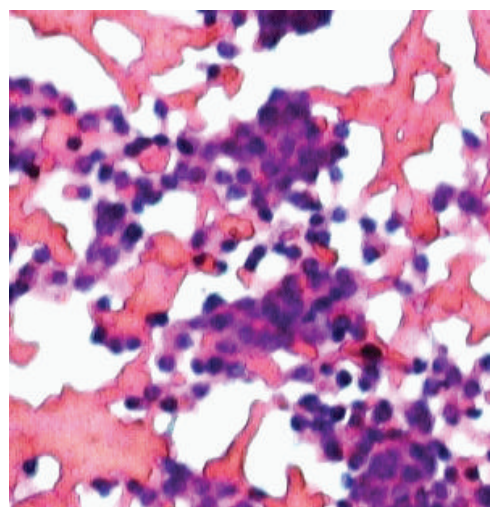

(c)

Figure 3: EUS (a), FNA (b), and cytological (c) images of the liver metastases.

Endoscopic ultrasound (EUS) is an extremely valuable tool in the diagnosis and management of these tumors. The important role of EUS in the detection of Pan-NETs was first described in 1992 [11]: EUS demonstrated a sensitivity of $82 \%$ and a specificity of $92 \%$ in the detection of islet cell tumors in patients whith previously undetected tumors by US and CT. Since then, EUS has been increasingly used in the localization of Pan-NETs [12]. EUS is particularly useful in the detection of smaller insulinomas. The average size of insulinomas at initial diagnosis is $6-10 \mathrm{~mm}$, with $90 \%$ of cases under $2 \mathrm{~cm}$ [13].

A recent study on 52 patients undergoing EUS for detection of a suspected insulinoma (based on clinical and laboratory findings) reported a sensitivity of $89.5 \%$ and accuracy of $83.7 \%$ based on surgical findings. The sensitivity of EUS for detection of lesions in pancreatic head, body, and tail was $92.6,78.9$, and $40.0 \%$, respectively [14].

The detection rates for pancreatic gastrinomas by EUS are similar to that of insulinomas, approximately $75-94 \%$ [15].

Pan-NETs may be pathologically evaluated by FNA during the EUS examination. Three recent studies reported sensitivities of $61-84 \%$ and overall accuracy of up to $92.5 \%$ of EUS-FNA in establishing the diagnosis of Pan-NETs [1618]. Additionally, FNA may detect and confirm the presence of malignant lymph nodes and liver metastases previously unseen on CT imaging [18, 19]. Recently, Lewis et al. [20] further confirmed in 52 patients the high accuracy of EUSFNA in preoperative assessment in MEN-1, and Barbe et al. [21] established a complementary role for MRI and EUS.

To the best of our knowledge, this is the first report where EUS-FNA has allowed not only the localization but also a complete diagnosis with pTNM staging of Pan-NETs.

\section{References}

[1] D. H. Schussheim, M. C. Skarulis, S. K. Agarwal et al., "Multiple endocrine neoplasia type 1: new clinical and basic findings," Trends in Endocrinology and Metabolism, vol. 12, no. 4, pp. 173-178, 2001.
[2] J. M. Glascock and S. E. Carty, "Multiple endocrine neoplasia type 1: fresh perspective on clinical features and penetrance," Surgical Oncology, vol. 11, no. 3, pp. 143-150, 2002.

[3] Q. Dong, L. V. Debelenko, S. C. Chandrasekharappa et al., "Loss of heterozygosity at 11q13: analysis of pituitary tumors, lung carcinoids, lipomas, and other uncommon tumors in subjects with familial multiple endocrine neoplasia type 1," Journal of Clinical Endocrinology and Metabolism, vol. 82, no. 5, pp. 1416-1420, 1997.

[4] D. Desai, L. A. McPherson, J. P. T. Higgins, and R. J. Weigel, "Genetic analysis of a papillary thyroid carcinoma in a patient with MEN 1," Annals of Surgical Oncology, vol. 8, no. 4, pp. 342-346, 2001.

[5] A. O. Vortmeyer, I. A. Lubensky, M. Skarulis et al., "Multiple endocrine neoplasia type 1: atypical presentation, clinical course, and genetic analysis of multiple tumors," Modern Pathology, vol. 12, no. 9, pp. 919-924, 1999.

[6] H. J. Kim, J. S. Park, C. S. Kim et al., "A case of multiple endocrine neoplasia type 1 combined with papillary thyroid carcinoma," Yonsei Medical Journal, vol. 49, no. 3, pp. 503-506, 2008.

[7] G. M. Doherty, J. A. Olson, M. M. Frisella, T. C. Lairmore, S. A. Wells, and J. A. Norton, "Lethality of multiple endocrine neoplasia type I," World Journal of Surgery, vol. 22, no. 6, pp. 581-587, 1998.

[8] T. C. Noone, J. Hosey, F. Zeynep, and R. C. Semelka, "Imaging and localization of islet-cell tumours of the pancreas on CT and MRI," Best Practice and Research, vol. 19, no. 2, pp. 195211, 2005.

[9] Y. M. Ku, S. S. Shin, C. H. Lee, and R. C. Semelka, "Magnetic resonance imaging of cystic and endocrine pancreatic neoplasms," Topics in Magnetic Resonance Imaging, vol. 20, no. 1, pp. 11-18, 2009.

[10] E. P. Tamm, E. E. Kim, and C. S. Ng, "Imaging of neuroendocrine tumors," Hematology/Oncology Clinics of North America, vol. 21, no. 3, pp. 409-432, 2007.

[11] T. Rösch, C. J. Lightdale, J. F. Botet et al., "Localization of pancreatic endocrine tumors by endoscopic ultrasonography," New England Journal of Medicine, vol. 326, no. 26, pp. 17211726, 1992.

[12] T. Ishikawa, A. Itoh, H. Kawashima et al., "Usefulness of EUS combined with contrast-enhancement in the differential diagnosis of malignant versus benign and preoperative localization 
of pancreatic endocrine tumors," Gastrointestinal Endoscopy, vol. 71, no. 6, pp. 951-959, 2010.

[13] G. Akerström and P. Hellman, "Surgery on neuroendocrine tumours," Best Practice \& Research Clinical Endocrinology \& Metabolism, vol. 21, no. 1, pp. 87-109, 2007.

[14] R. Sotoudehmanesh, A. Hedayat, N. Shirazian et al., "Endoscopic ultrasonography (EUS) in the localization of insulinoma," Endocrine, vol. 31, no. 3, pp. 238-241, 2007.

[15] A. M. McLean and P. D. Fairclough, "Endoscopic ultrasound in the localisation of pancreatic islet cell tumours," Best Practice and Research, vol. 19, no. 2, pp. 177-193, 2005.

[16] S. A. Pais, K. Mcgreevy, J. K. Leblanc et al., "Utility of EUSFNA in the diagnosis of pancreatic neuroendocrine tumors: correlation with histopathology in 76 patients," Gastrointestinal Endoscopy, vol. 65, no. 5, p. AB304, 2007.

[17] E. W. Holt, E. A. Macklin, and W. R. Brugge, "Variables affecting the accuracy of EUS-guided FNA in the diagnosis of focal pancreatic masses," Gastrointestinal Endoscopy, vol. 67, no. 5, pp. AB218-AB219, 2008.

[18] M. Atiq, M. S. Bhutani, M. Bektas et al., "EUS-FNA for pancreatic neuroendocrine tumors: a tertiary cancer center experience," Digestive Diseases and Sciences, vol. 57, no. 3, pp. 791-800, 2012.

[19] J. M. O’Neil, M. A. Al-Haddad, J. Leblanc et al., "Endoscopic ultrasound morphology features and initial detection of metastatic liver lesions from primary pancreatic adenocarcinoma and pancreatic neuroendocrine carcinoma," Gastrointestinal Endoscopy, vol. 67, no. 5, pp. AB217-AB218, 2008.

[20] M. A. Lewis, G. B. Thompson, and W.F. Young Jr, "Preoperative assessment of the pancreas in multiple endocrine neoplasia type 1," World Journal of Surgery, vol. 36, no. 6, pp. 1375-1381, 2012.

[21] C. Barbe, A. Murat, B. Dupas et al., "Magnetic resonance imaging versus endoscopic ultrasonography for the detection of pancreatic tumours in multiple endocrine neoplasia type 1," Digestive and Liver Disease, vol. 44, no. 3, pp. 228-234, 2012. 


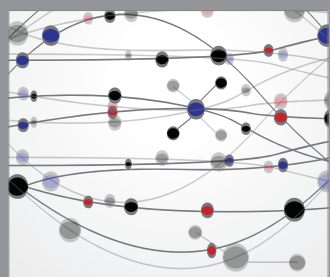

The Scientific World Journal
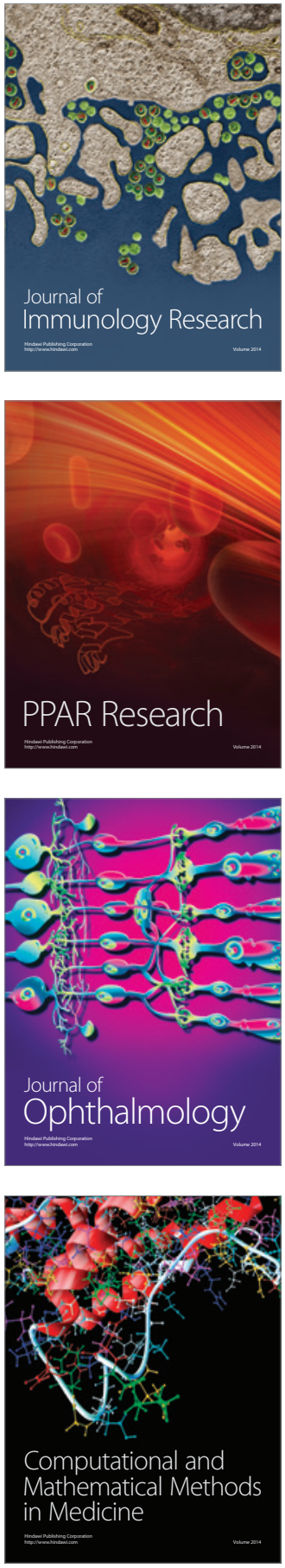

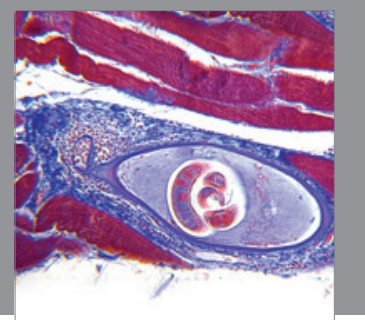

Gastroenterology

Research and Practice
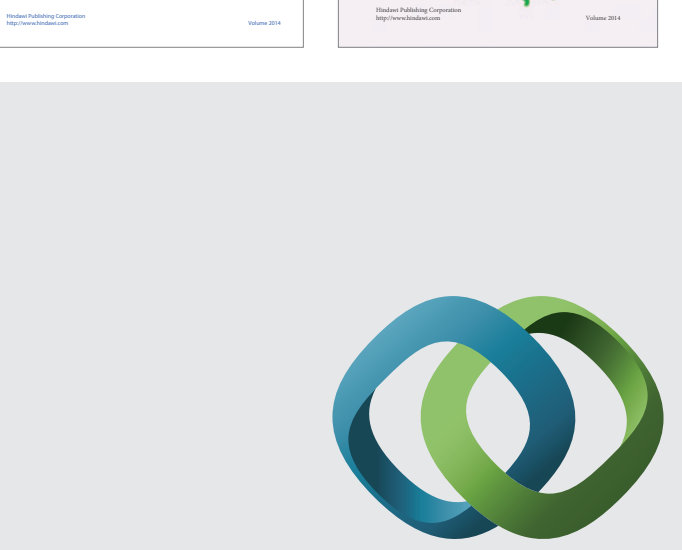

\section{Hindawi}

Submit your manuscripts at

http://www.hindawi.com
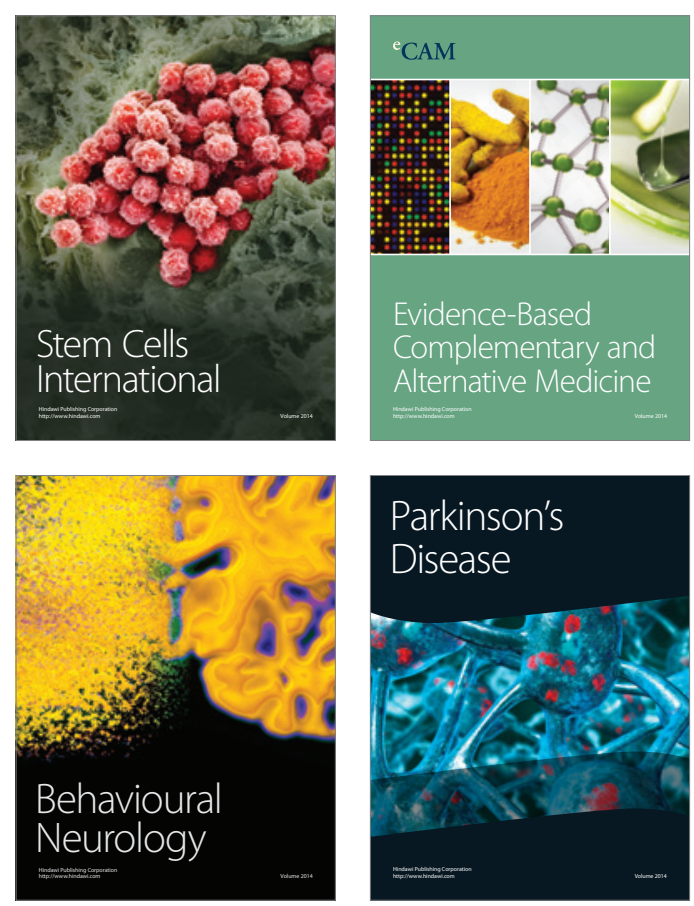

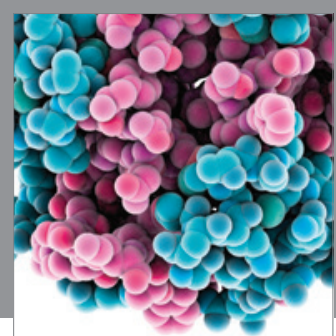

Journal of
Diabetes Research

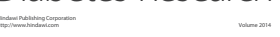

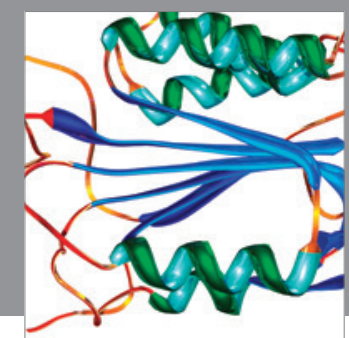

Disease Markers
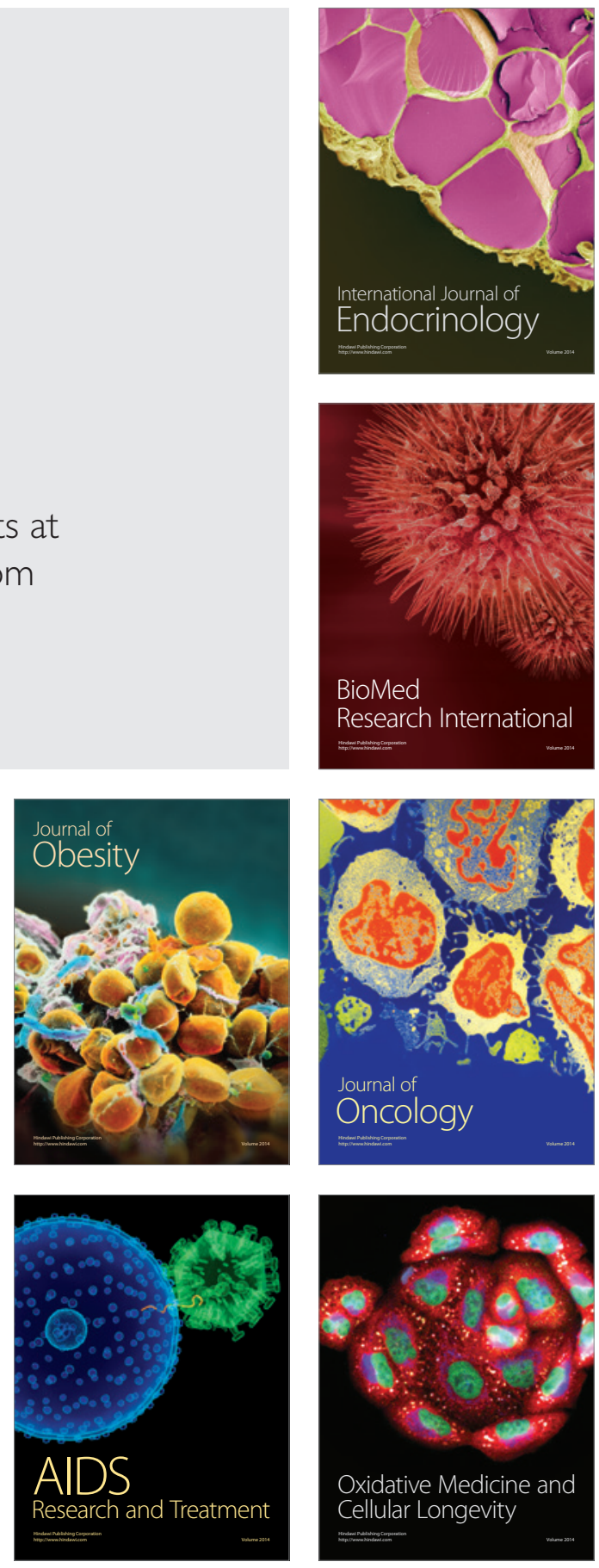